\title{
Oral Drug Delivery via Chronotherapy Approach: Need of the Day Jagadevappa Patil*
}

VT's Shivajirao S Jondhale College of Pharmacy, Asangaon-421 601, Shahapur, Thane, Maharashtra, India

*Corresponding author: Jagadevappa Patil, VT's Shivajirao S Jondhale College of Pharmacy, Asangaon-412 601, Thane, Maharashtra, India, Tel: +91 9448816812; Email: pharmajspatil@gmail.com

Received date: March 03, 2017; Accepted date: March 09, 2017; Published date: March 16, 2017

Copyright: (c) 2017 Patil J. This is an open-access article distributed under the terms of the Creative Commons Attribution License, which permits unrestricted use, distribution, and reproduction in any medium, provided the original author and source are credited.

\section{Introduction}

The drug delivery approach aimed to encounter the therapeutic requirements pertaining to particular pathological situations will be a newer research in formulation development area. Chronotherapy is a kind of treatment based on biological stimuli in drug therapy, and this has fetched a novel approach to the design of oral drug delivery systems. Several approaches are being exploited in the development of activated, pulsatile, controlled and programmed drug delivery strategies has exaggerated in recent years. Chronotherapeutics is the area related with drug delivery according to the intrinsic activities of a disease over a certain period of time. Chronotherapy deals with the treatment based on the human daily working cycle that corresponds to biological clock which the intention to enhance the therapeutic benefits and reduce the adverse effects. Chronotherapy mainly works by matching the timing of treatment with the intrinsic timing of illness. This method also refers to a kind of treatment approach in which in vivo drug availability is timed to match rhythms of disease, in order to improve therapeutic outcomes and reduce side effects. Optimal therapy can be achieved when the precise amount of drug is delivered to the correct target organ at the most suitable time. The drug release pattern will be varied based on the circadian rhythms and symptoms of a disease varied. The term chronothrapeutics is mainly novel in the field of drug delivery and in the treatment method. It is defined as an extensive term in which disease follow the circadian rhythm which undergoes the metabolic variations. Chronotehrapeutic strategies currently available control drug delivery by controlling the lag time independent environmental factors such as $\mathrm{pH}$, gastric motility, and biological enzymes. These systems are available both multiple and single units. This delivery approach is considered as a future of oral drug delivery because these are self-programmed and designed to release a specific drug at a specified rate at a particular time. These drug delivery devices are able to maintain required plasma concentrations when placed in the oral cavity and enhance the patient compliances by circumventing frequency of drug administration.

Chronic diseases, such as diabetes, hypertension, asthma, peptic ulcer, arthritis, etc., follow the body's circadian rhythm [1]. For instance, osteoarthritis prominent during the day and is most troublesome in the evenings and the pain usually peaks in the morning and decreases as the day progresses. Blood sugar level also enhances when sugar based food is consumed. Hypertension, angina, and chest pain, also follow a definite circadian rhythm. Epidemiologic studies have recognized the intensified morning-time risk of angina, myocardial infarction and stroke $[2,3]$.

\section{Types of Delivery Systems Available}

Several approaches such as time-controlled, pulsed, triggered and programmed drug delivery strategies have been established and comprehensively studied in recent years for chronopharmaceutical drug delivery. The important chronotherapeutic based oral pulsatile drug delivery systems are chronotherapeutic oral drug absorption system, Programmable oral drug absorption system, Dividable multiple action delivery system and spherical oral drug absorption system. Chronotherapeutic oral drug absorption system focuses on achieving sustaining and delaying in the drug action. This approach is used to develop the verapamil [4] an anti-arrhythmic drug and which is designed to release the verapamil tablet taken at night to maintain more concentration during early morning when the symptoms of arrhythmias are worsen [5]. The programmable oral drug absorption system is nothing but development of the tablet system within a capsule as a multi particulate system in order to control the drug release. The dividable multiple action delivery systems are meant to improve the drug efficacy by facilitating the tablet to be broken into two halves and release the drug at same rate thereby reducing the side effects. The spheroidal oral drug absorption system is a multiparticulate system that allows drug to be released in pulsatile bursts throughout the day. It mainly comprises spherical microbeads coated with polymers for controlled release [6].

\section{Conclusion}

Novel research and development in the field chronopharmacology successfully utilized the biological rhythms of human being in developing the chronotherapeutic drug delivery systems for drug therapy. In conventional sustained release dosage forms optimal clinical outcome cannot be achieved due to their common attitude of maintaining constant drug plasma concentrations all time. Based on variation of circadian with the display of symptoms of disease, drug release pattern from the dosage form should also vary over time. Many approaches have been utilized to fabricate time-controlled, pulsed, triggered and programmed drug delivery strategies in recent years. As the drug administration time in disease therapy showing substantial influence upon treatment success, chronotherapeutic drug delivery developments become an important research area. Hence, development of chronotherapeutic drug delivery devices helps in avoiding extensive first pass metabolism, chronotropic nature of the ailment and nocturnal drug administration thereby enhance the patient compliance and is the future of the oral drug delivery systems.

\section{References}

1. Ura J, Shirachi D, Ferrill M (1992) The chronotherapeutic approach to pharmaceutical treatment. California Pharmacist 23: 46-53.

2. Subal CB (2005) Chronotherapeutics: Optimising drug delivery.

3. Patil JS (2016) Chronotherapeutics: A Novel Approach in the Pharmacotherapy of Various Diseases. J Pharmacovigil 4: 3. 
Citation: Patil J (2017) Oral Drug

4. White WB, Mehrotra DV, Black HR, Fakouhi TD (1997) Effects of controlled onset extended release Verapamil on nocturnal blood pressureverapamil study group. Am J Cardiol 80: 469-474.

5. http://www.perrigo.com/
6. Pollock Dove C, Dong L, Wong P (2001) A new system to deliver a delayed bolus of liquid drug formulation. Proceed Intern Symp Control Rel Bioact Mater 28: 6033 . 